JIOM Nepal, Volume 41, Number 2, August 2019, page 35-39

\title{
Diagnostic Accuracy of Peritoneoscopy to Determine the Cause of Low Serum Ascites Albumin Gradient
}

\author{
'Pawan Parajuli, ${ }^{2}$ Ramesh S Bhandari, 'Rahul Pathak, 'Shashi Sharma, 'Prem K Khadga, 'Anurag Jha, \\ 'Rabin Hamal, 'Brindeswari Kafle \\ 1Department of Gastroenterology, Maharajgunj Medical Campus, Tribhuvan University Teaching Hospital, Institute of \\ Medicine, Maharajgunj, Kathmandu \\ ${ }^{2}$ Department of Gastointestinal and General Surgery, Maharajgunj Medical Campus, Tribhuvan University Teaching \\ Hospital, Institute of Medicine, Maharajgunj, Kathmandu
}

\section{Corresponding author:}

Pawan Parajuli, MBBS, MD

Department of Gastroenterology, Maharajgunj Medical Campus, Tribhuvan University Teaching Hospital, Institute of Medicine, Maharajgunj, Kathmandu

Email: pawanparajulee@gmail.com

Submitted: Apr 23, 2019

Accepted : Jun 20, 2019

\begin{abstract}
Introduction

Ascites, a common entity in practice of gastroenterology is pathophysiologically divided into high SAAG and Iow SAAG category, to rapidly classify, formulate a workup plan and expedite the diagnosis. The cause of low SAAG ascites is often due to local peritoneal cause e.g peritoneal tuberculosis, peritoneal carcinomatosis etc, mandating the need of peritoneoscopy for definitive diagnosis. This study aims to present the peritoneoscopy and peritoneal biopsy result of patients with low SAAG ascites of uncertain etiology.

\section{Methods}

Peritoneoscopy was prospectively performed in 12 patients with low SAAG ascites of unclear etiology. Patients with low SAAG ascites and willing to give consent for peritoneoscopy were enrolled in the study. Patients underwent laparoscopic peritoneoscopy under general anesthesia and appropriate biopsies were taken during the procedure for histopathological analysis.

\section{Results}

Of the twelve patients with low SAAG enrolled in the study, 3 (25\%) were male and 75\% (9) were female. The success rate of the procedure was $100 \%$ and there was no procedure related complications. Specific findings were seen in all patients undergoing peritoneoscopy. Of the twelve patients, 9 (75\%) patients has metastatic deposits in the peritoneum, $3(25 \%)$ had benign etiology, $2 / 3^{\text {rd }}(2)$ of whom had granulomatous deposits suggestive of tuberculosis and $1 / 3^{\text {rd }}(1)$ had extensive dense adhesions and peritoneal fibrosis. Primary focus was revealed (ovary) in only 1 patient undergoing peritoneoscopy.
\end{abstract}

\section{Conclusion}

Peritoneoscopy with simultaneous biopsy is safe, efficient and accurate diagnostic method due to its high diagnostic capacity and low complication rate in selected patients who have low SAAG ascites of uncertain etiology.

Keywords: Ascites, peritoneal carcinomatosis, peritoneoscopy

\section{INTRODUCTION}

A scites is pathological accumulation of fluid in the peritoneal cavity. Ascites is a common occurrence in the clinical practice of gastroenterology. Effective treatment of ascites is largely dependent on identification of the underlying cause.

Pathophysiologically ascites has been classified into two broad groups viz. portal hypertension related, most of which is caused by the cirrhosis of liver and the other group which is not related to portal hypertension e.g. causes related to the peritoneum (local cause) like tuberculosis, peritoneal malignancy etc. The convenient and easiest way to identify these broad causes of ascites is by means of estimating serum ascites and albumin gradient (SAAG) value. Portal hypertension related ascites presents with SAAG value $>1.1$ and those not related to portal hypertension presents with SAAG value $<1.1$ which is 
taken as cut off. ${ }^{1}$ Owing to different pathophysiology the practice of classifying the ascites as exudate and transudate has been abandoned unlike in pleural fluid analysis which is broadly categorized as either exudative or transudate. ${ }^{2,3}$

Low SAAG ascites are caused by disease of the peritoneum (local cause). The main causes of these low SAAG ascites are peritoneal malignancies and tuberculosis as shown in most of the studies. Therefore expedited workup of low SAAG ascites is mandated owing to the serious diseases associated with it. On one hand tuberculosis is a curable entity and on the other peritoneal malignancy is incurable and have favorable and ominous prognosis respectively.

Due to close proximity intra-abdominal tumors and infections seed to peritoneum in early stages. ${ }^{4}$ These intraperitoneal seedings can be detected by modern imaging techniques and by means of peritoneoscopy. ${ }^{5,6}$ Peritoneoscopy has been successfully used for diagnostic purposes in exudative ascites with uncertain etiology. ${ }^{3}$ The procedure of peritoneoscopy is relatively easy to perform and can even be performed in endoscopy unit with sedation anesthesia. The need for peritoneoscopy has decreased nowadays but due to its high diagnostic value, is still regarded as the "gold standard" method for diagnosis of exudative (low SAAG) ascites with unknown etiology. 9.10

In this we study we prospectively aimed to look into the peritoneoscopy and peritoneoscopic biopsy results of patients presenting to our hospital with low SAAG ascites of unknown etiology.

\section{METHODS}

Patients presenting with ascites to the department of gastroenterology of Tribhuvan University Teaching Hospital, Kathmandu were evaluated and those which yielded a low SAAG on evaluation and the cause of which was not known with routine investigations of ascitic fluid and imaging modalities were prepared for peritoneoscopy after thoroughly explaining the procedure. The consent for the procedure was taken in the written form from either the patient or the attendant. After the preanesthetic checkup by the anesthesiologist for fitness for the procedure, the patients were subjected to peritoneoscopy performed by the expert gastrointestinal surgical team. Post procedure the patients were thoroughly monitored and then shifted to the inpatient department. Relevant data was collected in prespecified proforma.

Patients with new onset ascites were admitted to the inpatient ward of Gastroenterology Department of Tribhuvan University Teaching Hospital, Kathmandu. Routine blood investigations including liver function tests, renal function tests, coagulation profile and viral markers for hepatitis A, B, C was obtained. Complete
Patients with ascites admitted for evaluation

$$
\downarrow
$$

Routine investigations of ascitic fluid performed $\downarrow$

Serum ascites albumin gradient (SAAG) calculated

$$
\downarrow
$$

Patients with low SAAG ascites selected and subjected to battery of investigation

$\downarrow$

CEA, CA-125, CEA, exfoliative malignant cytology, CECT abdomen

$\downarrow$

Inconclusive by above means were subjected to peritoneoscopy

\section{Figure 1. Flow diagram showing the methodology of the study}

hemogram was also obtained. Imaging in the form of chest X-ray and routine ultrasound scan of the abdomen was also performed. This was followed by diagnostic paracentesis of ascitic fluid whereby cell counts including differential count, protein, albumin and sugar concentration of the fluid was obtained including Adenosine deaminase (ADA) assay. The fluid was also subjected to analysis for exfoliative malignant cytology. SAAG was then calculated from the value of serum albumin and ascitic fluid albumin concentration. Patients with value of SAAG less than $1.1 \mathrm{~g} / \mathrm{dl}$ then underwent computed tomography scan of the abdomen to see if there is any clue to the cause of their low SAAG ascites. If the cause of the ascites was evident from the CT scan workup was stopped and appropriate therapy was initiated. However those patients with inconclusive CT scan of the abdomen were subjected to peritoneoscopy after discussing with patients in detail about the procedure and nature of illness. The methodology undertaken is depicted in the flowchart below.

The inclusion criteria comprised patients with low SAAG $(<1.1)$ ascites where disease modality could not be ascertained by other means including standard imaging.

Subjects with high SAAG $(>1.1)$ with history of acute abdomen and abdominal trauma were not included. Similarly, subjects not fit for surgery were not included.

As a part of preparation of the patients for the procedure, patients were kept nil per oral the night before peritoneoscopy. After obtaining written consent, intravenous line was maintained and third generation cephalosporin (Ceftriaxone $1 \mathrm{gm}$ ) was 
Table 1. Demographic and laboratory parameters

\begin{tabular}{lc}
\hline \multicolumn{1}{c}{ Parameters } & Mean \\
\hline Age & $47.66 \pm 15.68$ \\
Hemoglobin & $11.27 \pm 2.15$ \\
Total Protein & $63.83 \pm 6.99$ \\
Albumin & $32.25 \pm 6.9$ \\
Ascitic Fluid Protein & $44.8 \pm 6.84$ \\
Ascitic Fluid Albumin & $24.8 \pm 6.1$ \\
Mean SAAG & $7.45 \pm 2.06$ \\
\hline
\end{tabular}

given before the procedure. All the procedure was done under general anaesthesia.

Under aseptic conditions two coats of painting by $10 \%$ povidone iodine and draping was done. Pneumoperitoneum was created by open technique through $1 \mathrm{~cm}$ long vertical incision just below the umbilicus. $\mathrm{CO} 2$ was used to create pneumoperitoneum and pressure of 8-12 $\mathrm{mm} \mathrm{Hg}$ was maintained. The infraumbilical port was used for laparoscope (camera port) (Karl storz $10 \mathrm{~mm}$ 30 degree). One or more other ports was used as required. Placement of other working ports $(5 \mathrm{~mm})$ was determined by suspected underlying pathology or possible biopsy site. The infraumbilical port was closed in layers with vicryl 2.0 for fascia and ethilon 2.0 for skin. Other working $5 \mathrm{~mm}$ ports were closed in single layers using ethilon 2.0. Patient were shifted to recovery room and then to ward. Dressing of the wound site was done on alternate day basis. Suture was removed after 10 days.

The study is approved by the Institutional Review committee of Institute of Medicine, TUTH. Written informed consent was obtained from all patients prior to enrollment

\section{RESULTS}

The mean age of patients was $47.66 \pm 15.68$ years. Among the patients enrolled in the study 3 out of $12(25 \%)$ were males and remaining were females. Six $(50 \%)$ patients were less than 50 years of age. $3(25 \%)$ patients had benign etiology of their ascites whereas $9(75 \%)$ had malignancy as a cause of their ascites. Of the benign etiology two (two-third) had granulomatous inflammation and one had fibrotic reaction in the peritoneum.

Demographic and laboratory characteristics of the fourteen patients are presented in table 1

The distribution of patients according to etiology is

Table 2. Distribution of patients according to etiology

\begin{tabular}{lc}
\hline \multicolumn{1}{c}{ Etiology } & Numbers (\%) \\
\hline Peritoneal carcinomatosis & $9(75 \%)$ \\
Tubercular peritonitis & $2(16.66 \%)$ \\
Peritoneal fibrosis & $1(8.34 \%)$ \\
\hline
\end{tabular}

shown in table 2. and the presenting complaints of the patients are shown in table 3.

Of all the twelve patients with low SAAG ascites, nine had evidence of peritoneal carcinomatosis as a cause of their ascites. Of all the patients with peritoneal carcinomatosis (9), the primary focus was evident as ovary in one $(11.11 \%)$ patient whereas in the rest the clue to the primary was not known (table $4)$. Malignancy was seen in $44 \%$ (4) of patients in less than 50 years of age and $56 \%$ (5) were seen in more than 50 years age group. Of all the patients with malignancy, two (22\%) were males and seven (78\%) were females.

Of all the 11 cases with peritoneal deposits, four (36.4\%) patients were reported to have tubercles out of which $50 \%$ turned out to be tuberculosis and the rest peritoneal metastases. $29 \%$ of cases had elevated ADA levels above $30 \mathrm{U} / \mathrm{L}$ of which half were tuberculosis and half were metastatic peritoneal deposits.

The various histopathological diagnosis reported from peritoneal biopsies is shown in table 5

Of all, eleven patients were found to have peritoneal deposits, out of which six $(54.5 \%)$ cases were reported as having peritoneal tubercles out of which only two (33\%) patients turned out to be having granulomatous inflammation consistent with tuberculosis and the rest were histologically proven metastatic adenocarcinoma. Adenosine deaminase was elevated (above 30U/L) in 4(33\%) out of 12 patients of which $50 \%$ had granulomatous inflammation consistent with tuberculosis and the rest had metastatic malignancy.

\section{DISCUSSION}

Ascites of unknown etiology is defined as ascites in which the cause cannot be determined after conventional laboratory tests and further radiologic testing. ${ }^{4}$ It is this ascites of unknown etiology which poses a major diagnostic problem to the clinicians. The causes of ascites of unknown etiology varies considerably with the geographic locale and ethnic origin. Peritoneal carcinomatosis and tuberculosis constitute a majority of these cases of ascites of unknown etiology. ${ }^{11,12}$

The primary function of the peritoneal membrane is to protect the abdominal viscera and to limit the diffusion of inflammation. Peritoneum being in

Table 3. Presenting symptoms of patients

\begin{tabular}{lc}
\hline \multicolumn{1}{c}{ Etiology } & Numbers (\%) \\
\hline Abdominal distension & $12(100 \%)$ \\
Pain abdomen & $12(100 \%)$ \\
Fever & $4(33.33 \%)$ \\
Weight loss & $5(41.66 \%)$ \\
\hline
\end{tabular}


Table 4. Primary focus search in patients with peritoneal carcinomatosis

\begin{tabular}{lc}
\hline \multicolumn{1}{c}{ Primary focus } & Numbers (\%) \\
\hline Ovary & $1(10 \%)$ \\
No primary focus detected & $9(90 \%)$ \\
\hline
\end{tabular}

close proximity to the abdominal organs, tumors and infections may spread to the the peritoneum in early stages. ${ }^{4}$ Previously, diagnosis of peritoneal diseases relied on surgical methods. In the present days by using advanced radiologic methods such as computerized tomography (CT) and magnetic resonance imaging, these lesions may be readily diagnosed, while the rest are identifiable with peritoneoscopy.

Peritoneoscopy is a less invasive method and has low risk compared to surgery; ${ }^{4}$ however, undesired injuries may occur sometimes namely hemorrhage, perforation and air embolism. ${ }^{13,14}$ Generally, it is a reliable method when performed by experienced specialists. Nowadays, peritoneal biopsies guided by ultrasound and CT have become quite frequent. These methods demonstrate less complications compared with peritoneoscopy, and they have quite successful outcomes. ${ }^{15-18}$

The majority of patients in our series had peritoneal carcinomatosis as the most common cause of their ascites, which is in consistence with the previous literatures. ${ }^{4,19}$ The majority of cases with malignant ascites were more than 50 years of age consistent with the fact that malignancy tends to be commoner in older age groups.

Peritoneal carcinomatosis is followed by tuberculosis as the cause of ascites in our series which is again in harmony with the studies done in the past. ${ }^{4,19}$ Though tuberculosis is highly endemic in our part of the world, it did not top the list of the etiology. The possible reason could be due to improvements in the imaging modalities, increasing use of colonoscopy and to the widespread availability and adoption of testing of serum ADA (adenosine deaminase) in the ascitic fluid sample based on which the diagnosis of tuberculosis is established and treated.

If ascites is present, paracentesis is the least invasive procedure but ascitic fluid analysis is not usually of specific diagnostic value for tuberculosis because acid fast bacilli (AFB) are rarely found in ascitic fluid and culture for $M$. tuberculosis is often negative and time consuming. ${ }^{20-22}$ Laparotomy was recommended as the procedure of choice in the diagnosis of tubercular peritonitis but complications and mortality of this procedure are higher than peritoneoscopy. ${ }^{12,20}$ On the other hand peritoneoscopy is a safe, simple, well tolerated and cheap method for accurately diagnosing intra-abdominal disease.

Of the nine patients with malignant etiology as the
Table 5. Histopathological diagnosis from peritoneal biopsies

\begin{tabular}{|c|c|}
\hline Histopathological diagnosis & Numbers (\%) \\
\hline $\begin{array}{l}\text { Metastatic carcinoma } \\
\text { Granulomatous inflammation } \\
\text { Extensive fibrosis with } \\
\text { mesothelial proliferation }\end{array}$ & $\begin{array}{c}9(75 \%) \\
2(16.66 \%) \\
1(8.34 \%)\end{array}$ \\
\hline
\end{tabular}

cause of peritoneal disease, the primary was found as ovary in only one patient, which was missed in CT, however in the rest there was no clue to the primary.

Morphology of the peritoneal deposit is an important clue to the diagnosis. Smaller and uniformly distributed peritoneal deposits are more consistent with benign etiology compared to deposits in peritoneal carcinomatosis which tends to be larger, of different sizes and morphology and non uniformly distributed than tubercular deposits. In our series six cases were reported as having tubercles owing to uniformity of size, out of which only two (33\%) cases turned out to be tuberculosis and $67 \%$ turned out to be carcinomatosis. All other non uniformly distributed deposits of varying sizes were peritoneal metastases as in previous reports.

The typical appearance of peritoneum in tuberculous peritonitis was described as "turbot skin appearance" since the peritoneal surfaces were studded with homogenous miliary tubercles not bigger than a pinhead and resembling the skin of aTurbot. ${ }^{23}$ Although turbot skin appearance was highly suggestive of tuberculous peritonitis, in $67 \%$ of cases malignancy was misdiagnosed as tuberculous peritonitis since uniform sized nodules were present and they were evenly distributed over the peritoneum. This problem has been experienced by others as well. 12,20,21

There are other causes of nodular peritoneal deposits viz crohns disease, sarcoidosis, starch peritonitis, coccidiodiomycosis. ${ }^{12,20,21}$ These are however rarer causes of peritoneal deposits and in our series none of the peritoneal biopsies were conforming to the above mentioned diagnosis reminiscent of the fact that these are rare etiologies and the occurrence depends on the geographical location and commonality of the disease.

The clinical presentation of all the patients were similar in having pain abdomen and distension irrespective of the etiology (benign/malignant). However, fever was present in patients with both the diagnosis, though it was present in all of the patients with tuberculosis but only in $2(22 \%)$ cases of peritoneal malignancy, possibly malignancy related fever.

All patients subjected to peritoneoscopy confirmed to certain diagnosis after visualization and peritoneal biopsies. Majority of patients $(75 \%)$ had peritoneal carcinomatosis followed by tuberculosis (16.66\%). One $(8.34 \%)$ patient had extensive fibrosis in the 
peritoneum with mesothelial proliferation contributing to ascites.

All cases with peritonoscopic biopsies in this study concluded with certain diagnosis. Compatible with the literature, the most frequent diagnosis was carcinoma peritonitis, followed by tuberculous peritonitis, which is frequently seen in this geographic location.

In this study the Peritoneoscopic appearance was found more accurate than other diagnostic modalities. The diagnosis of tuberculous peritonitis and peritoneal carcinomatosis could be made on the basis of typical peritoneal membrane appearances supported by histologic demonstration of caseating or noncaseating granuloma and tumorous cells in the peritoneal biopsies. All of the patients demonstrating the granuloma and treated with anti-tuberculosis therapy (ATT) recovered. Therefore, peritoneoscopy with simultaneous biopsy is the ideal and the most accurate diagnostic modality in the diagnosis of patients with low SAAG ascites.

\section{CONCLUSION}

Peritoneoscopy with direct visualization and peritoneoscopic biopsies in conjuction has high diagnostic yield and is highly accurate in the evaluation of low SAAG ascites of unknown etiology. Peritoneoscopy is a safe and cost effective diagnostic modality in expert hands and provides definitive diagnosis in majority of patients, when almost all non-invasive investigations remain inconclusive. This study demonstrated that the peritonoscopic biopsy is still efficient in selected exudative ascites patients with uncertain etiology.

\section{CONFLICT OF INTEREST}

None declared.

\section{REFERENCES}

1. Ruddock JC. Peritoneoscopy. Surg Gynnecol Obstet 1937; 65: 623.

2. Vargas $C$, Jeffers $L J$, Bernstein $D$, Reddy $R$, Munnangi S, Behar S, Parker et al. Diagnostic Laparoscopy: A 5 years experience in a hepatology training program. Am J Gastroenterol 1995; 90:1258.

3. Brady PG, Peebles M, Goldschmid S. Role of Laparoscopy in the evaluation of patients with suspected hepatic or peritoneal malignancy. Gastrointest Endos 1991;37:27.

4. Abayli B, Gencdal G. A Single center experience: The diagnostic role of peritoneoscopy in patients with exudative ascites. Journal of Laparoendoscopic and Advanced Surgical Techniques 2018;00:1-4. DOI: 10.1089/lap.2018.0274

5. Chu CM, Lin SM, Peng SM, Wu CS, Liaw YF. The role of laparoscopy in the evaluation of ascites of unknown origin. Gastrointest Endosc 1994; 40:285-9.
6. Apaydin B, Paksoy M, Bilir M, Zengin K, Saribeyoglu K, Taskin M. Value of diagnostic laparoscopy in tuberculous peritonitis. Eur J Surg 1999; 165:158-63.

7. Body W. P. Laparoscopy in ascites and peritoneal diseases. In: Sivak M. V., ed., Gastroenterologic Endoscopy. W. B. Saunders, Philadelphia, 1987; 1109-19.

8. Lightdale C. Indications, contraindications and complications of laparoscopy. In; Sivak M. V., ed., Gastroenterologic Endoscopy. W. B. Saunders, Philadelphia, 1987; 1030-44.

9. Sugarbaker PH, Yan H, Grazi RV, Shmookler BM. Early localized peritoneal mesothelioma as an incidental finding at laparoscopy. Report of a case and implications re- garding natural history of the disease. Cancer 2000;89:1279-1284.

10. Han CM, Lee CL, Huang KG, Chu CM, Lin SM, Wang CJ, et al. Diagnostic laparoscopy in ascites of unknown origin: 20-year experience. Chang Gung Med J 2008;31:378382.

11. Inadomi JM, Kapur S, Kinkhabwala M, Cello JP, et al. The laparoscopic evaluation of ascites. Gastrointest Endosc Clin N Am 2001;11:79-91.

12. Menzies RI, Fizgerald JM, Mulpeter K. Laparoscopic diagnosis of ascites in Lesotho. Br Med J (Clin Res Ed) 1985; 291:473-475.

13. Hall TJ, Donaldson DR, Brennam TG. The value of laparoscopy under local anaesthesia in 250 medical and surgical patients. Br J Surg 1980;67:751-3.

14. Nord HJ. Biopsy diagnosis of cirrhosis blind percutaneous versus guided direct vision technique-a review. Gastrintest Endoscop 1982;28:102.

15. Layfield LJ, Gopez EV. Percutaneous image-guided fineneedle aspiration of peritoneal lesions. Diagn Cytopathol 2003;28:6-12.

16. Sistrom $C L$, Abbitt $P L$, Feldman PS. Ultrasound guidance for biopsy of omental abnormalities. J Clin Ultrasound 1992;20:27-31.

17. Souza FF, Mortele' KJ, Cibas ES, Erturk SM, Silverman SG. Predictive value of percutaneous imaging-guided bi- opsy of peritoneal and omental masses: Results in 111 patients. AJR 2009;192:131-136.

18. Pombo F, Rodriguez E, Martin R, Lago M. CT-guided core-needle biopsy in omental pathology. Acta Radiol 1997;38:978-981.

19. Nasir Hassan Luck, Anwaar A. Khan, Altaf Alam, Arshad Kamal Butt, Farzana Shafquat. Role of Laparoscopy in the diagnosis of Low Serum Ascites Albumin Gradient. J Pak Med Asso 2007;57:33-34

20. Reddy K.R, Diprima R. E, Raskin J.B. et al. Tuberculous peritonitis. Laparoscopic diagnosis of uncommon disease in the United States. Gastrointest. Endosc. $1988 ; 34 ; 422-6$

21. Wolfe J.H.N., Behn A.r. \& Jackson B. T. Tuberculous peritonitis and role of diagnostic laparoscopy. Lancet 1979; i:852-3.

22. Bender M. D. Disease of the peritoneum, mesentery and diaphragm. In: Slesinger M.H., Fordtran, J. S. eds, Gastrointestinal Disease. W. B. saunders, Philadelphia, 1989;1932-67.

23. Colakoglu S, Unal S, Ergun $Y$, Sandicki M, keskin B \&Yilmaz I. Peritoneal tuberculosis in Cukurova area. Cuk. Uni. Tip. Fak. Derg. 1988; 34 (suppl.):876-8. 